\title{
Erratum to: Estimating leaf chlorophyll contents by combining multiple spectral indices with an artificial neural network
}

\author{
Pudong Liu ${ }^{1,2,3} \cdot$ Runhe Shi ${ }^{1,2,3,4} \cdot$ Wei Gao ${ }^{1,2,3,4,5}$
}

Published online: 15 September 2017

(C) Springer-Verlag GmbH Germany 2017

Erratum to: Earth Sci Inform

https://doi.org/10.1007/s12145-017-0319-1

The original version of this article, unfortunately, contained an error.

The above article originally published with an error within the funding section. Originally reading "National Key Research and Development Program of China (No. 2016YFC1201305)", this should instead have read "National Key Research and Development Program of China (No. 2016YFC1302602)."

The online version of the original article can be found at https://oi.org/ 10.1007/s12145-017-0319-1

Runhe Shi

rhshi@geo.ecnu.edu.cn

1 Key Laboratory of Geographic Information Science, Ministry of Education, East China Normal University, Shanghai 200241, China

2 School of Geographic Sciences, East China Normal University, Shanghai 200241, China

3 Joint Laboratory for Environment Remote Sensing and Data Assimilation, East China Normal University \& Institute of Remote Sensing and Digital Earth Chinese Academy of Sciences, Shanghai 200241, China

4 Joint Research Institute for New Energy and the Environment, East China Normal University and Colorado State University, Shanghai 200062, China

5 Department of Ecosystem Science and Sustainability, Colorado State University, Fort Collins, CO 80523, USA 